\title{
BMJ Open Evaluation of risk mitigation measures for people with substance use disorders to address the dual public health crises of COVID-19 and overdose in British Columbia: a mixed-method study protocol
}

Bohdan Nosyk (1) , ${ }^{1,2}$ Amanda Slaunwhite, ${ }^{3}$ Karen Urbanoski, ${ }^{4,5}$ Natt Hongdilokkul, ${ }^{6}$ Heather Palis, ${ }^{3}$ Kurt Lock, ${ }^{3}$ Jeong E Min, ${ }^{2}$ Bin Zhao, ${ }^{3}$ Kiffer G Card,,${ }^{4,5}$ Brittany Barker (D) , ${ }^{7,8}$ Louise Meilleur, ${ }^{7}$ Charlene Burmeister, ${ }^{9}$ Erica Thomson, ${ }^{10}$ Phoenix Beck-McGreevy, ${ }^{10}$ Bernie Pauly (i) ${ }^{4,11}$

To cite: Nosyk B, Slaunwhite A, Urbanoski K, et al. Evaluation of risk mitigation measures for people with substance use disorders to address the dual public health crises of COVID-19 and overdose in British Columbia: a mixed-method study protocol. BMJ Open 2021;11:e048353. doi:10.1136/ bmjopen-2020-048353

- Prepublication history and additional supplemental material for this paper are available online. To view these files, please visit the journal online (http://dx.doi.org/10.1136/ bmjopen-2020-048353).

Received 23 December 2020 Revised 27 April 2021 Accepted 05 May 2021

Check for updates

(C) Author(s) (or their employer(s)) 2021. Re-use permitted under CC BY-NC. No commercial re-use. See rights and permissions. Published by BMJ.

For numbered affiliations see end of article.

Correspondence to Dr Bohdan Nosyk; bnosyk@sfu.ca

\section{ABSTRACT}

Introduction The COVID-19 pandemic was preceded by an ongoing overdose crisis and linked to escalating drug overdose deaths in British Columbia (BC). At the outset of these dual public health emergencies, the $\mathrm{BC}$ government announced interim Risk Mitigation Guidance (RMG) that permitted prescribing medication alternatives to substances, including opioids, alcohol, stimulants and benzodiazepines, an intervention sometimes referred to as 'safe supply'. This protocol outlines the approach for a study of the implementation of RMG and its impacts on COVID-19 infection, drug-related and systemic harms, continuity of care for people with substance use disorder (SUD), as well as their behavioural, psychosocial and wellbeing outcomes.

Methods and analysis We conducted a parallel mixedmethod study that involved both analysis of populationlevel administrative health data and primary data collection, including a 10-week longitudinal observational study (target $n=200$ ), a cross-sectional survey (target $n=200$ ) and qualitative interviews (target $n=60$ ). We implemented a participatory approach to this evaluation, partnering with people with lived or living expertise of drug use, and researchers and public health decision-makers across the province. Linked population-level administrative databases will analyse data from a cohort of $\mathrm{BC}$ residents with an indication of SUD between 1996 and 2020. We will execute high-dimensional propensity score matching and marginal structural modelling to construct a control group and to assess the impact of RMG dispensation receipt on a collaboratively determined set of primary and secondary outcomes.

Ethics and dissemination Study activities were developed to adhere to the Tri-Council Policy Statement: Ethical Conduct for Research Involving Humans, recommended COVID-19 research practices, and guided by the Truth and Reconciliation Commission's Calls to Action for public health, data governance and research ethics related to Indigenous people. Results will be

\section{Strengths and limitations of this study}

- Using a mixed-methodological approach to evaluate outcomes and implementation, this study constitutes the first formal evaluation of Risk Mitigation Guidance, initially developed for COVID-19 and the overdose crisis and intended to provide regulated pharmaceutical alternatives to a toxic illicit drug supply.

- Combining longitudinal analysis of a robust population-level administrative health dataset and targeted primary data collection, including a 10week longitudinal observational study, a crosssectional survey and qualitative interviews, this study will capture the dynamic nature of the evolving context around the intervention.

- Ongoing engagement with provincial policymakers, clinicians and people with lived or living expertise of drug use will ensure this study fully captures and characterises the intervention as it evolves.

- We will conduct high-dimensional propensity score matching and marginal structural modelling to construct a control group and to assess the impact of 'safe supply' on a collaboratively determined set of primary and secondary outcomes.

- The possibility of unmeasured confounding remains. However, our proposed analytical approach provides the best possible opportunity to obtain a causal effect of the programme on the primary and secondary outcomes, using several approaches to adjust for baseline confounding and a parallel analysis of longitudinal and cross-sectional observational data.

disseminated incrementally, on an ongoing basis, through the consortium established for this study, then published in peer-reviewed journals. 


\section{INTRODUCTION}

Like other jurisdictions across the globe, British Columbia (BC) Canada has implemented extensive public health measures to reduce the transmission of SARS-CoV-2 (COVID-19). However, Canada is also facing an escalating overdose epidemic. ${ }^{1-4}$ The COVID-19 pandemic was preceded by an ongoing public health emergency in BC, declared in April 2016 as a result of the rapid rise in overdose deaths. BC reported 1547 illicit drug overdose deaths in $2018,{ }^{5}$ accounting for over a third of overdose deaths in Canada despite being home to just $12 \%$ of Canadian residents. ${ }^{6}$ The fourfold increase in overdose deaths since 2012 has largely been attributed to the contamination of the illicit drug supply with fentanyl and fentanyl analogues. The most recent BC Coroner's report detected fentanyl in $87 \%$ of all drug-related deaths. Among those who have died from overdose, many had sought treatment in the past and returned to using illicit drugs, underlining the need to both strengthen the quality of care and to expand the set of options to treat or reduce harm resulting from licit and illicit substance use. ${ }^{7}$

The declaration of a BC-wide public health emergency in April 2016 prompted a coordinated and multifaceted public health response. ${ }^{8}$ This included measures to increase accessibility to opioid agonist treatment $(\mathrm{OAT})^{9-14}$ and the expansion of harm reduction services, including drug checking services, naloxone distribution and supervised consumption and overdose prevention sites. These measures appeared to have resulted in some progress in reducing illicit drug overdose deaths in 2019 where the number of overdose deaths declined to 984 . Since March 2020, however, there have been substantial increases in overdose deaths, with over 170 persons dying per month from May 2020 to July 2020, the highest monthly figures ever recorded in the province. ${ }^{15}$ First Nations in BC have also experienced an increase in overdose deaths with an overdose death rate 5.6 times that of other residents of BC for the first 5 months of $2020 .^{16}$

At the intersection of these dual public health emergencies are a number of challenging issues that increase the risk of substance-related and systemic harms, including heightened risk of overdose due to restrictions in access to medical care, social services and harm reduction services, ${ }^{16}{ }^{17}$ contradictory public health messaging that encourages physical distancing while discouraging using drugs alone $\mathrm{e}^{18} 19$ and an increasingly toxic illicit drug supply. ${ }^{20-22}$ People who use drugs are at heightened risk of COVID-19 infection due to high rates of compounding comorbidities and exposure to conditions where implementing physical distancing and personal hygiene protocols is challenging. ${ }^{23-25}$

Federal and provincial governments have implemented policies to counteract potential secondary effects of the public health response to COVID-19, including allowing pharmacists to renew prescriptions ${ }^{26}$ issuing temporary exemptions for OAT prescriptions, ${ }^{27}{ }^{28}$ increased use and promotion of telehealth and medication delivery, ${ }^{29-31}$ and an exemption to the Controlled Drugs and Substances
Act for prescribers, pharmacists and patients. ${ }^{28}$ Provincial and municipal governments have also introduced programmes to increase access to supportive housing, through the purchase of hotels and reappropriation of other public facilities with increased access to harm reduction interventions, ${ }^{32-34}$ and announced expansions of inpatient detoxification services in some settings to manage the increased demand for these services ${ }^{35} 36$ and to address the high number of overdose deaths that occur within single-room occupancy hotels and other private dwellings. ${ }^{15} 37$

\section{Implementation of Risk Mitigation Guidance (RMG)}

On 26 March 2020, the BC government announced new interim RMG to (1) prevent the spread of COVID-19 by enabling physical distancing and self-isolation among people who use substances ('substance' is used in this article to be inclusive of illicit and licit substances, including alcohol and tobacco; 'drug' is used to refer to illicit drugs, excluding alcohol and tobacco, and emphasising the unique experiences and risks of illicit drug use); and (2) as harm reduction response to the increasingly toxic drug supply as a result of the pandemic. ${ }^{38}$ The RMG permits prescribing of opioids, stimulants, benzodiazepines, as well as medication alternatives to alcohol and nicotine use, for persons who are COVID-19-positive or at high risk of infection, combined with a high likelihood of withdrawal, overdose or other substance-related and systemic harms. According to the guidelines, in principle, the availability of medication alternatives to illicit drugs would also reduce the risk of exposure to and transmission of COVID-19 by reducing the need to seek purchase or acquisition of illicit drugs.

Prior randomised controlled trials (RCTs) provide indirect support for the impetus supporting RMG implementation. Among people with opioid use disorders who were unable to be retained in treatment with oral opioids like methadone or buprenorphine, a number of RCTs have demonstrated that injectable diacetylmorphine and hydromorphone are safe, effective and cost-effective treatments. ${ }^{39-41}$ In the absence of widespread expansion of injectable OAT, hydromorphone tablet distribution programmes have been implemented in recent years to provide alternatives to the toxic drug supply and to reduce the risk of fatal overdose. ${ }^{42} 3$

Although more evidence is needed, there is emerging support for the RMG as it pertains to substances other than opioids. A recent meta-analysis of RCTs has concluded that dextroamphetamine can have a clinically significant benefit in promoting abstinence among people with cocaine use disorder. ${ }^{44}$ For amphetamines, promising results have been demonstrated in reducing craving and use and improving retention, where robust doses of psychostimulants have been prescribed. ${ }^{45-47}$ Among people with alcohol use disorder, a number of RCTs have demonstrated that anticonvulsants such as carbamazepine $e^{48-53}$ and gabapentin ${ }^{545}$ and clonidine (alpha-2 adrenergic agonist) ${ }^{56}$ are as safe and effective 
as benzodiazepines for the management of alcohol withdrawal symptoms. Long-acting benzodiazepines such as clonazepam have been used to safely and effectively taper patients off of shorter-acting benzodiazepines such as alprazolam (Xanax).$^{58}$ RCTs are ongoing to test the effectiveness of different pharmacological approaches to managing benzodiazepine use disorder and discontinuation, bearing in mind side effects, benefits and risks. ${ }^{59}$

Aforementioned studies have been conducted as evaluations of pharmacological treatment options, with the explicit goal of cessation of illicit drug use or abstinence. Furthermore, they have been generated from highly controlled studies, where medications have been consumed under the supervision of healthcare providers to promote adherence and to monitor adverse events. It is likely that the positioning of the RMG as a means of reducing substance-related harm, rather than an explicit form of pharmacological treatment, and its delivery in real-world settings may draw clients who are not directly comparable to persons recruited in the aforementioned trials conducted in controlled conditions with strict eligibility criteria (for instance, screening out individuals with moderate or severe mental health conditions, which are common in people with substance use disorders (SUDs) ). ${ }^{60}$ Furthermore, the reach of the intervention across the province and the fidelity to the initial recommendations stated in the RMG are unknown. The RMG lists only oral opioids (ie, tablet hydromorphone and M-eslon) which have not been the subject of the same rigorous evaluations of safety or effectiveness for the treatment of opioid use disorder in comparison to injectable treatments. In summary, while prior RCTs ${ }^{40}$ have informed the opioid options listed in the RMG, the results of these trials are not immediately comparable to the medications listed in the RMG.

The Guidance outlines eligibility based on individual risk of COVID-19 infection, those confirmed COVID-19 positive or those with a suspected case (eg, symptomatic and self-isolating); those with a history of ongoing active substance use (opioids, stimulants, alcohol, benzodiazepines or tobacco); and those that are deemed at high risk of withdrawal, overdose, craving or other harms related to substance use. Youth aged $<19$ years may be eligible if there is informed consent by the client or parent to receive this intervention and additional education is provided. It is noted that prescribers should first offer alternative options (eg, OAT). Clinical assessment includes current and past substance use, history of overdose, comorbid mental and physical conditions, prescribed medication(s) and access to a prescriber. For persons who do not have a primary care provider (general practitioner/ family doctor or nurse practitioner), or in cases where the primary care provider declines the service, rapid access addiction services and OAT clinics have accepted new patients seeking medications through the RMG. Table 1 provides a full list of the medications identified in the RMG. The medications are accessed free of charge to BC residents under the province's PharmaCare programme. ${ }^{38}$

\section{Study objectives}

There is a critical need for comprehensive assessment of the implementation and impacts of the RMG on COVID-19 transmission and risk of overdose in BC. We anticipate uptake to be uneven across the province, given the variation across prescribers in education and resources, as well as variation in local and regional resources, services and support. Prescribers and allied healthcare professionals are tasked with developing models of service delivery for RMG while simultaneously adapting to new clinical guidance and strains on their practice in addressing the COVID-19 pandemic. In BC as elsewhere, the system of services and support for people who use substances is complex, and services are provided in a variety of settings, including community health centres, specialised residential and outpatient treatment, OAT clinics, and a mixture of peer-run and professionally led overdose prevention/supervised consumption sites, among others.

This protocol outlines the approach for a mixedmethod study of the implementation of RMG and its impacts on COVID-19 infection and substance-related harms, including overdose. Specific objectives were to

1. Determine the impact of RMG on COVID-19 infection, non-fatal/fatal overdose, all-cause mortality and continuity of care for SUD and other concurrent health conditions.

2. Determine the impact of RMG on the uptake of public health measures to reduce the spread of COVID-19, as well as other behavioural and psychosocial outcomes among people who use substances.

3. Identify individual, interpersonal and systemic barriers and facilitators to RMG implementation based on programme uptake from the perspectives of people who use substances, prescribers and other health service providers.

This study evaluates the RMG as a policy intervention introduced into BC's health system in March 2020, in the context of dual public health emergencies. In terms of the clinical intervention supported by this policy, our focus is on the receipt of an RMG prescription of pharmaceutical alternatives for opioids, alcohol, stimulants, benzodiazepines and other drugs. It should be noted that referral to managed alcohol programmes is also mentioned in the RMG as an alternative for providers to consider in supporting people who have alcohol use disorders. There is separate operational guidance for Managed Alcohol Programs during COVID-19 in BC (https://www.bccsu.ca/wp-content/uploads/2020/10/ Operational-Guidance-Managed-Alcohol.pdf). So, for pragmatic and methodological reasons, this protocol is limited to RMG prescription-based interventions for alcohol and drugs.

A parallel evaluation by the Canadian Managed Alcohol Study team (www.cmaps.ca) is ongoing to evaluate the emergence of managed alcohol programmes as part of the response to COVID-19. 


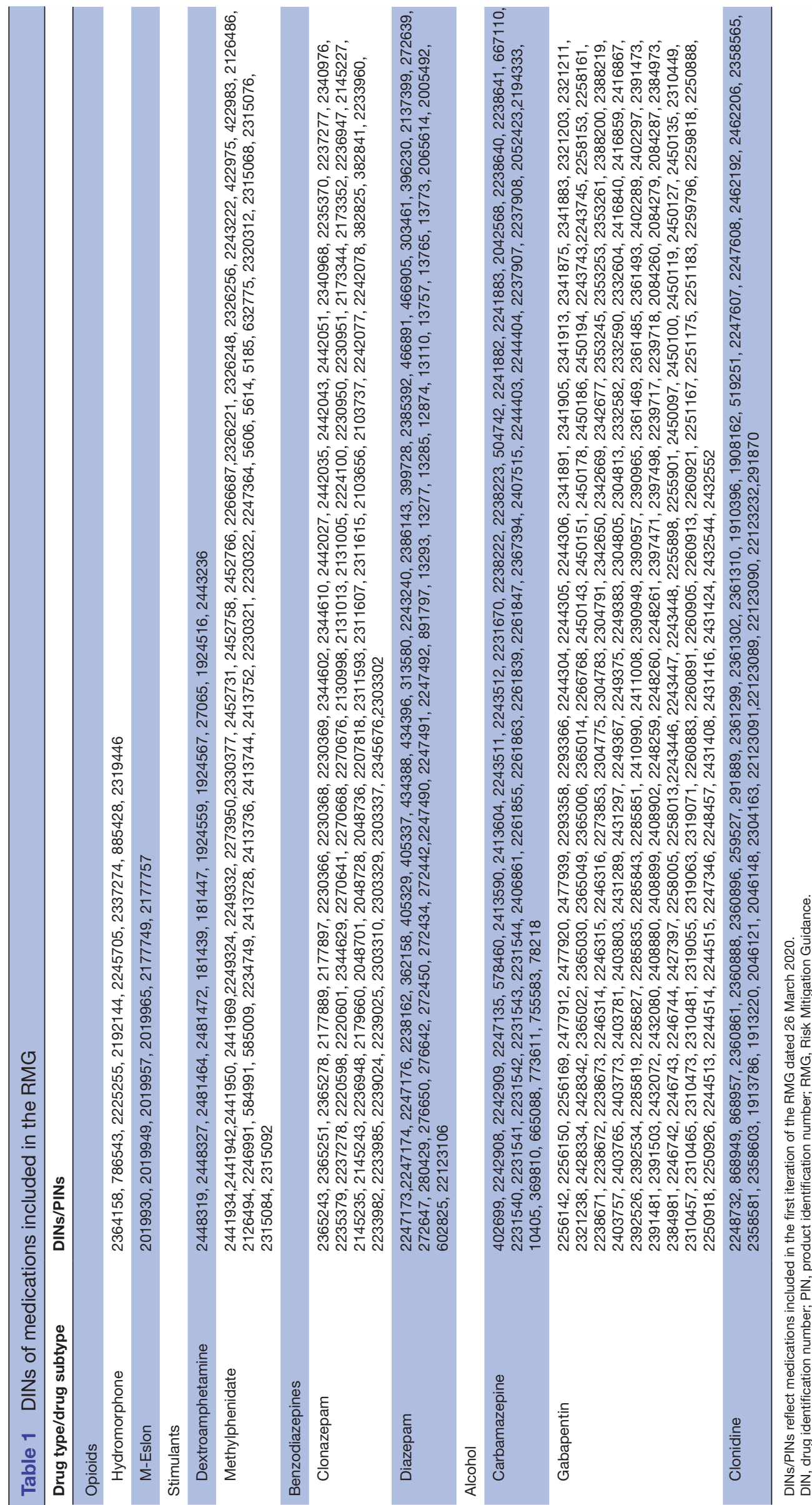




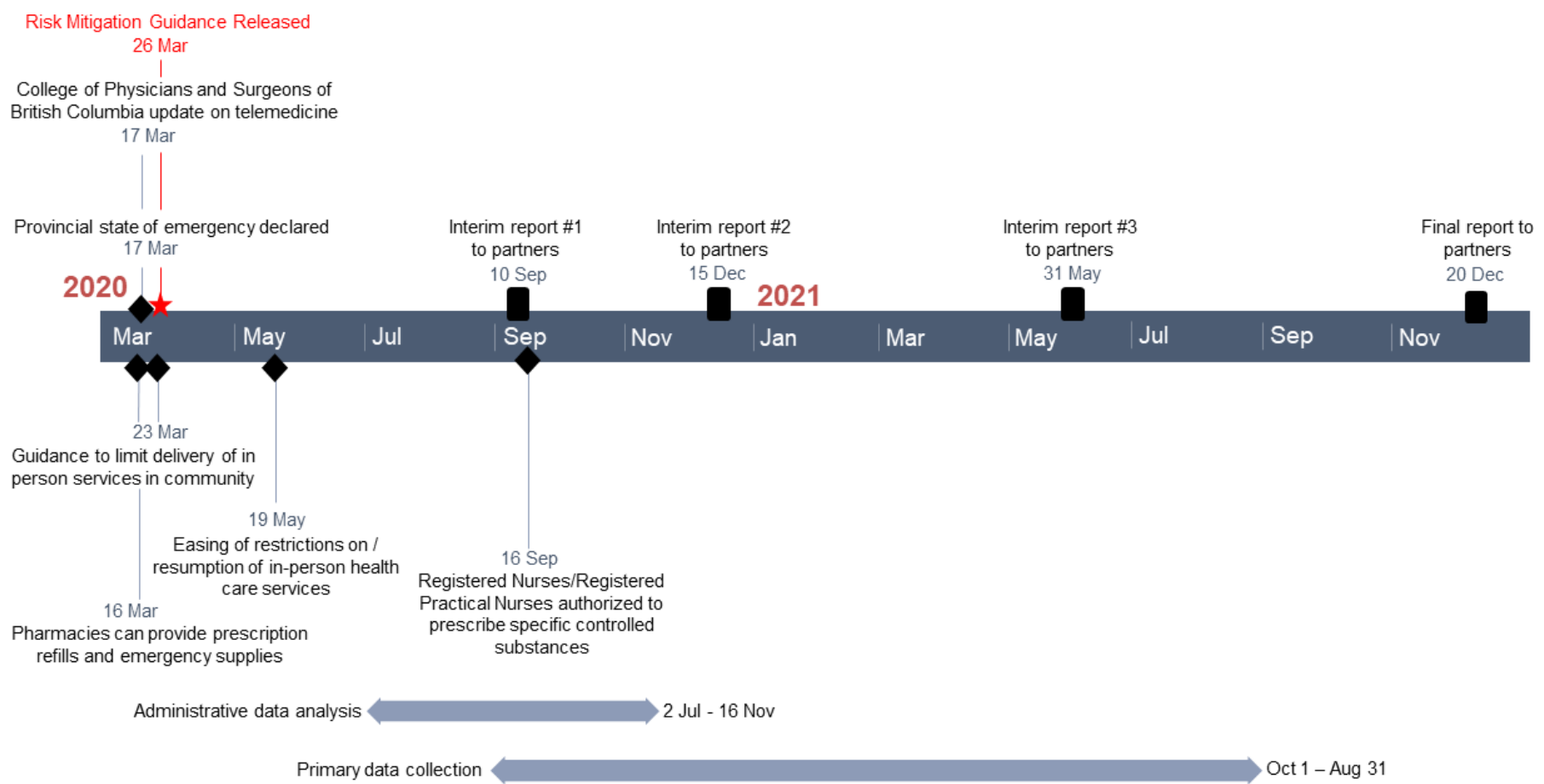

Figure 1 Study timeline.

\section{METHODS AND ANALYSIS \\ Study design}

We will achieve the aforementioned objectives through a parallel mixed-method study, with convergent collection and analysis of quantitative and qualitative data. ${ }^{6162}$ The full study involves both primary data collection, including a longitudinal (10weeks) observational study, crosssectional survey, and qualitative interviews, as well as analysis of administrative health data. These components are described in turn. A timeline for the study is provided in figure 1.

\section{Patient and public involvement}

The study uses an integrated knowledge translation approach, with stakeholders variously involved in all phases of the research. Study governance is provided by a core team of scientific leads who report to members of two groups/organisations of people with lived or living expertise (PWLLE) of drug use, service providers, health planners, policymakers and research experts; the provincial drug user organisation (BC/Yukon Association of Drug War Survivors (BCYADWS)) expressly approached two of the coauthors (KU and $\mathrm{BP}$ ) to initiate research on the evaluation of the RMG guidance on release. Separate working groups have been convened to support the analysis of administrative health data and primary data collection components of the study. The primary data collection component is explicitly participatory, with deep involvement of PWLLE, including Indigenous PWLLE as well as Indigenous and First Nations community members involved in designing data collection tools, recruitment processes, analysis and knowledge translation with opportunities for mutual exchange and learning through biweekly meetings. Peer research associates (ie,
PWLLE) will be hired in collaboration with drug user groups/organisations. Core team members include representatives from the provincial health authority that oversees healthcare to First Nations communities (ie, the First Nations Health Authority (FNHA)), a peer network embedded within the BC Centre for Disease Control (Professionals for Ethical Engagement of Peers), and a network of independent, autonomous grass-roots drug user groups from across the province (BCYADWS).

\section{Theoretical framework}

The study is grounded in the Consolidated Framework for Implementation Research (CFIR), ${ }^{6364}$ extended to include a concurrent evaluation of outcomes. ${ }^{65}$ The CFIR has been used to guide implementation research across a broad range of intervention types and settings, including substance use and public health. It consists of 39 theoretical concepts or constructs grouped into five domains capturing determinants affecting implementation of an intervention at the organisational level. The five domains include (1) characteristics of individuals receiving and delivering the intervention (eg, sociodemographic characteristics, knowledge and skills); (2) characteristics of the intervention (eg, ease of access, eligibility and models of delivery); (3) process of implementation (eg, leadership and training); (4) inner setting (eg, policies, organisational culture, space and staffing); and (5) outer setting (eg, funding, policies and laws). Attention is given throughout the analyses to align each of these implementation constructs with primary and secondary outcomes (described further). While not all constructs are addressed in every component of the study, the CFIR framework will inform primary surveys and qualitative interviews to better understand what was actually done 


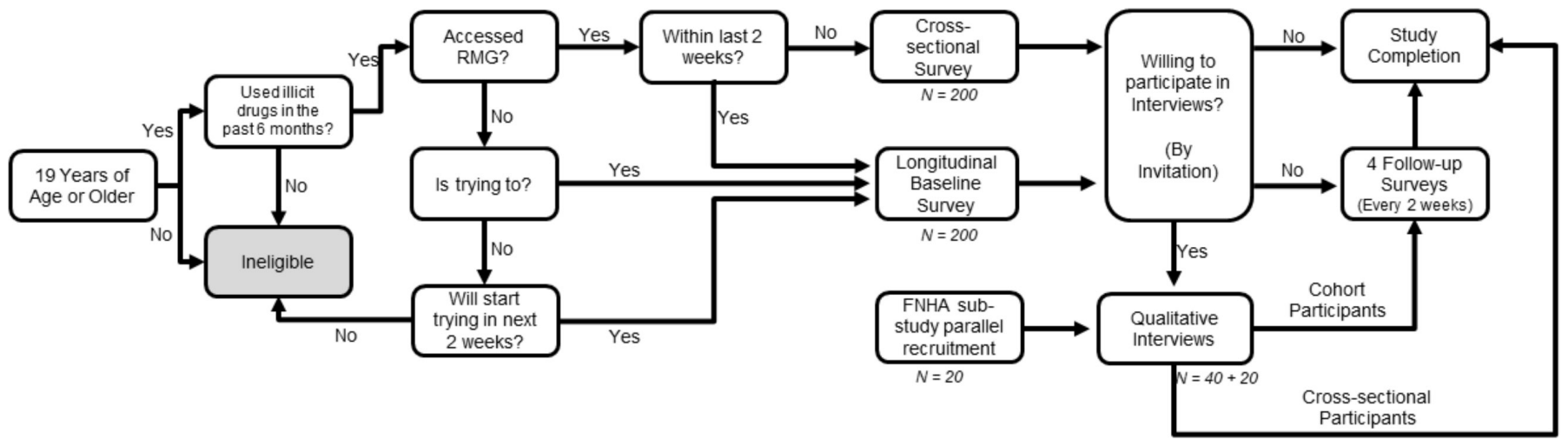

Figure 2 Flowchart for recruitment into the longitudinal and cross-sectional observational study. RMG, risk mitigation guidance.

in each of the five domains with attention to specific constructs within each domain. This approach is part of the overall mixed-method approach to the study in which administrative data and primary data collection are being conducted in parallel with a view to the primary data providing insight and assisting with interpretation of administrative data.

\section{Study population}

The study population includes people who use drugs and alcohol, and health service providers who work with people who use drugs and alcohol in BC. Eligibility criteria vary somewhat across study components (described further) but are designed to collectively capture the primary stakeholders of the RMG intervention. Recruitment will feature oversampling of individuals with Indigenous ancestry and ensure a proportional distribution of rural and urban respondents.

\section{Longitudinal and cross-sectional observational study: recruitment and data collection}

People who use drugs will be recruited to complete surveys as part of an observational study, with longitudinal and cross-sectional arms (figure 2). Posters and online advertisements (with a toll-free phone number and study email address) will be shared by community organisations, drug user groups, harm reduction and health service providers. In-person recruitment will also be done in selected venues by peer research assistants and other members of the research team (supported by an approved COVID-19 safety plan for research). Snowball sampling will be used (ie, participants will be invited to share the contact information with others who may be interested). Prospective participants will be screened for eligibility. Eligibility criteria include being 19 years old or older and having used illicit drugs in the past 6 months. Those who received their first RMG prescription within the past 2 weeks or who are currently attempting or intending to access a RMG prescription in the next 2 weeks (at time of recruitment) will be eligible for the longitudinal study (target $\mathrm{n}=200$ ). They will be asked to complete the survey five times at 2-week intervals (follow-up period $=10$ weeks). Given the variation in access to providers and programmes that support RMG, it is expected that the longitudinal sample will include a mix of respondents who were and were not successful in accessing RMG during the 10-week follow-up period. Those who received their first RMG prescription longer than 2 weeks ago (at time of recruitment) will be eligible for the cross-sectional arm of the study and will complete the survey once only (target $n=200$ ). Surveys can be completed online, by phone or in person.

Recruitment strategies will be monitored carefully and adapted as needed to ensure that the final samples will permit analytical comparisons by access to RMG. For both the cross-sectional and longitudinal survey arms, attention will be paid during recruitment to ensure representation by sex/gender, rural/urban setting, substance type (opioids, stimulants, benzodiazepines, and alcohol), and Indigenous ancestry. All eligibility and sampling criteria are determined by self-report. It is a limitation of this study that, given the sampling strategy (eg, use of convenience and snowball sampling), the survey will not generate a random sample, nor will it be possible to control for a design effect to adjust for non-random sampling.

The surveys (baseline and follow-up) will assess access to RMG prescriptions, ability to implement public health guidelines for COVID-19 (eg, physical distancing, selfisolation, mask-wearing, access to testing and treatment), substance use and related harms, sources of income (including acquisition crime and sex work), healthrelated quality of life (5-Level Euro-Qol 5 Dimension) ${ }^{66} 67$ and mental health (Patient Health Questionnaire-2 and Generalized Anxiety Disorder 2-item). ${ }^{689}$ These domains have been selected in accordance with the RMG and through consultation with PWLLE on anticipated impacts. With the exception of bespoke items on COVID19 , measures have demonstrated reliability and validity in use with people who use substances.

\section{Qualitative interviews: recruitment and data collection}

A subsample of survey participants (people who use drugs) will be recruited for qualitative interviews $(n=40)$. Interview participants will be purposively sampled to obtain representation by sex/gender, rural/urban and drug 
type, as well as whether the participant was successful in accessing an RMG prescription. Lead by FNHA, an additional 20 interviews will be conducted with people who identify as Indigenous (First Nations, Inuit or Métis) for a total of 60 interviews with people who use drugs.

Interview guides have been developed to align with the CFIR, assessing facilitators and barriers to implementation across the five domains of the CFIR.$^{63}$ Focus will be placed on capturing experiences of access to RMG and aspects of delivery models. For example, barriers and facilitators to accessing safer supply, how and where safe supply is accessed and experiences with accessing safer supply, as well as impacts of obtaining access. Facilitators and barriers will be explored across and within CFIR domains to create a comprehensive understanding of implementation and implications for programming. FNHA investigators and Indigenous stakeholders (eg, PWLLE and First Nations community members) will interview a subsample of Indigenous participants to yield culturally relevant findings on RMG implementation and to inform FNHA policy and programming and support for First Nation communities, particularly rural and remote communities. In BC, Indigenous people who use drugs have been disproportionately impacted by the overdose emergency ${ }^{71}$ and experience elevated and intersecting barriers accessing healthcare services (eg, stigma and discrimination, geography).

Additional interviews will be conducted with service providers and heath planners $(\mathrm{n}=40)$ who are involved in RMG delivery and those who opt not to participate in RMG delivery. We will purposively recruit providers from different disciplinary backgrounds: prescribers (physicians and nurse practitioners), pharmacists, nurses and harm reduction workers. Interview guides will be constructed as previously mentioned using CFIR interview templates, structured to assess facilitators and barriers to implementation across the five domains. This will yield information on provider knowledge of and level of comfort with the RMG, as well as information needs, and personal and organisational goals, philosophies and models for service delivery.

\section{Analysis of administrative health data: data source and linkage}

Population-based analyses will be conducted using linked administrative health datasets that capture healthcare use across the province. In BC, medically necessary healthcare is covered by universal health insurance, through a single-payer, government-administered plan that is available to all registered residents of the province. The plan covers most hospitalisations, acute and emergency care, primary care, and some mental health and substance use services. Prescription pharmaceuticals are covered for selected medical diagnoses and populations; however, records for all dispensations are retained regardless of the public or private health insurance payer. Health benefits for First Nations in BC are administered by the FNHA.
Datasets capturing these different sectors of healthcare will be accessed and linked for analysis (table 2).

The provincial SUDs cohort is an administrative database that captures all BC residents with an indication of SUD since $1996 .{ }^{72}{ }^{73}$ The cohort is identified using 11 linked population-level administrative databases, capturing provincial health insurance plan registration, physician billing records, hospitalisations, medication dispensations, health services delivered within provincial prisons, emergency department visits, perinatal services for all provincial births, receipt of housing and income assistance, mortality and cause of death including toxicology analysis. Linkage is achieved via probabilistic matching by the BC Ministry of Health and delivered to the investigators stripped of identifying information.

In addition to the provincial SUD cohort, records from the COVID-19 cohort will be accessed for this study. The COVID-19 cohort, held at the BC Centre for Disease Control, includes medication dispensations, mortality and COVID-19 PCR testing records. For both cohorts, linkage across datasets is accomplished at the record level via unique personal health numbers. Both cohorts will be refreshed to capture data through the end of 2020.

\section{Outcomes and implementation processes}

Primary and secondary outcomes and implementation process indicators are defined in table 3 , along with their data source (whether captured in the longitudinal/cross-sectional survey, qualitative interviews or administrative health data). Primary outcomes are fatal/non-fatal overdose and COVID-19 infection. These outcomes were identified explicitly in the RMG and/or are expected (based on supporting literature and/or discussion with stakeholders) to be most directly impacted by the RMG. Secondary outcomes capture effects that are hypothesised to be intermediates on the causal pathway between RMG exposure and primary outcomes (eg, continuity of care), or that may be expected to shift given RMG exposure but that are influenced by a greater array of factors external to the RMG (eg, health-related quality of life, mental health and illicit substance use). Secondary outcomes were typically identified as important factors to consider by stakeholders (including PWLLE). They are included here to support broader exploration of the effects of programmes that offer people who use substances a 'safer supply'. Finally, a set of implementation process indicators has been defined to align with the domains of the CFIR.

\section{RMG exposure}

Receipt of RMG prescriptions will be elicited via self-report in primary data collection and identified through administrative health data on medication dispensations. Relevant to the definition of RMG exposure in the administrative health data, we found that medications dispensed through RMG were already being used for therapeutic purposes and thus had drug identification numbers (DINs) assigned to them. When RMG was implemented, new DINs were not assigned. Physicians were instructed to add free-form codes to the directions for use field on the RMG prescriptions to differentiate them 


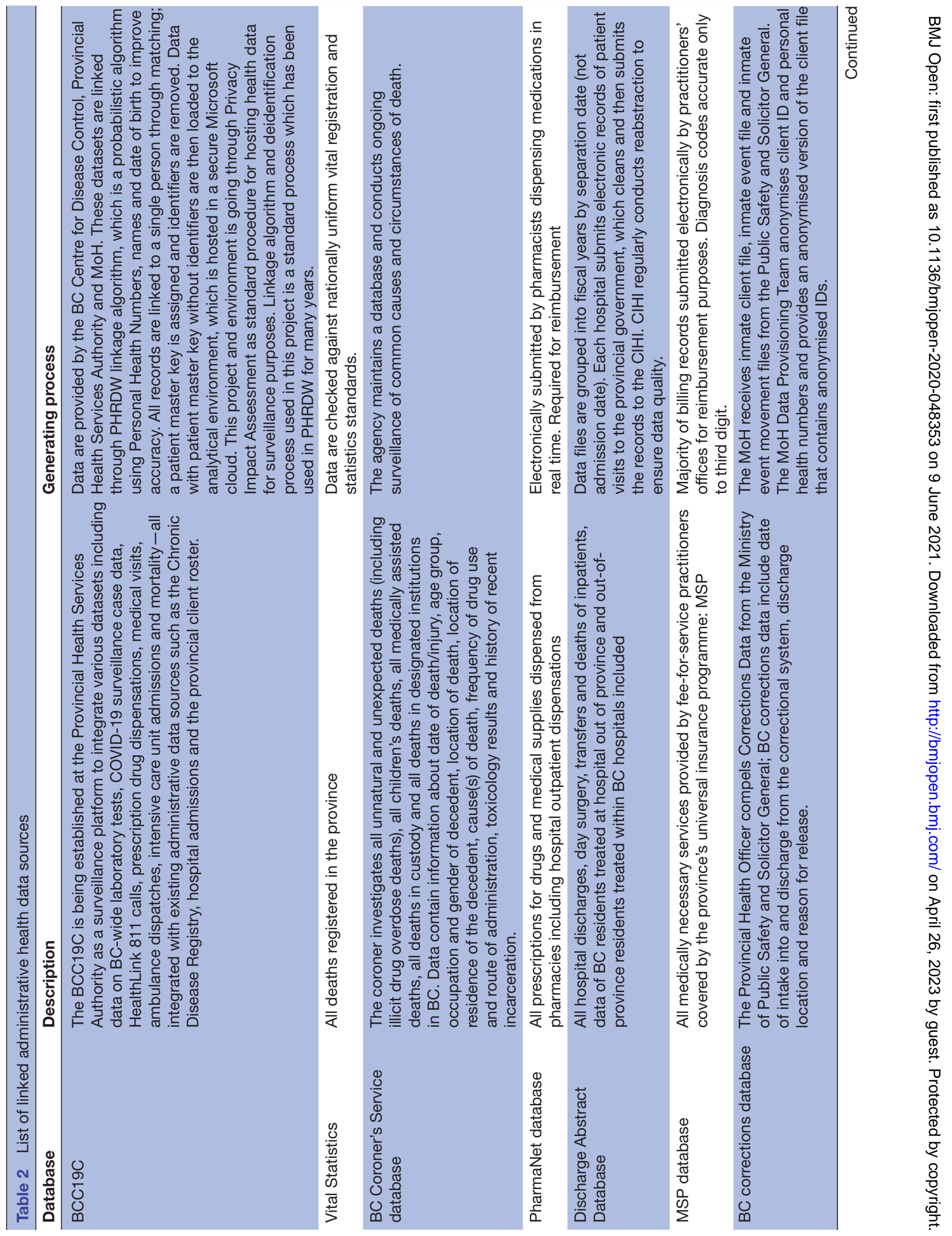




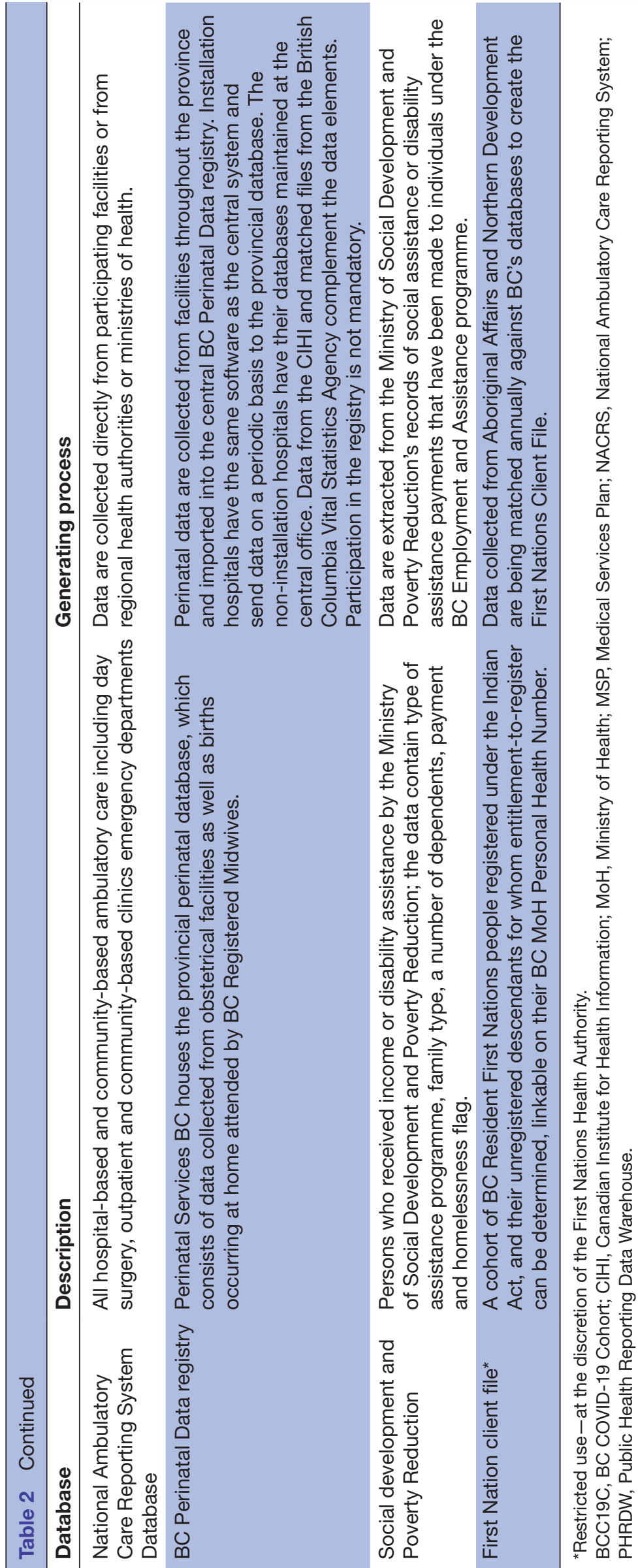


Table 3 Primary and secondary outcomes and implementation process indicators

\begin{tabular}{|c|c|c|}
\hline Outcome & Rationale and description & Data sources \\
\hline \multicolumn{3}{|l|}{ Primary outcomes } \\
\hline Incidence of SARS-CoV-2 infection & According to the initial impetus for the RMG & Public Health Laboratory system \\
\hline Incidence of fatal overdose* & $\begin{array}{l}\text { Coroner's record with laboratory testing } \\
\text { confirming presence of a range of substances. } \\
\text { Concordance of these classifications will be } \\
\text { confirmed with linked vital statistics data. }\end{array}$ & $\begin{array}{l}\text { BC Coroner's service database, } \mathrm{BC} \\
\text { vital statistics database }\end{array}$ \\
\hline Incidence of non-fatal overdose* & $\begin{array}{l}\text { ED visit or hospitalisation for drug-related } \\
\text { causes }\end{array}$ & NACRS and DAD databases \\
\hline
\end{tabular}

Secondary outcomes

Incidence of all-cause mortality We will consider all-cause mortality to account for the uncertainty in attribution of illicit drugs in mortality records and otherwise acknowledge the secondary role of drug use in deaths due to other causes.

Incidence of all-cause acute care visits to hospital, ED $\dagger$

\section{OAT retention among PWOUD}

We will consider all-cause acute care visits to account for the uncertainty in attribution of illicit drugs in health administrative records and otherwise acknowledge the secondary role of drug use in causes of other hospitalisations.

We will consider variations of definitions, including (but not limited to) OAT episode discontinuations§, missed doses ? and sustained disengagement..*

Retention/continuity of care for other
chronic medical conditions

Uptake of COVID-19 protective measures

Health-related quality of life
Mental health

Mental health

\begin{abstract}
Substance use and related harms
\end{abstract}
Income source
The medical conditions under consideration will include HCV, mental health disorders and potentially other conditions cited in consultations with community stakeholders.

Differences in ability to maintain physical distancing and self-isolate when needed, based on RMG exposure

Differential change (over 10 weeks) in scores
on the EQ-5D-5L, based on RMG exposure

Differential change (over 10 weeks) in scores on the Patient Health Questionnaire-2/

Generalized Anxiety Disorder 2-item, based on RMG exposure

Differential change (over 10 weeks) in use of illicit opioids, stimulants, and benzodiazepines (without a prescription), and in binge drinking, based on RMG exposure
BC Coroner's service database, BC vital statistics database
PharmaNet, MSP and DAD

Longitudinal survey data

Longitudinal survey data

Longitudinal survey data

Longitudinal survey data

Differential change (over 10 weeks) in sex work Longitudinal survey data and acquisition crime as income source, based on RMG exposure

\section{Implementation Outcomes}

Number of people who receive an RMG prescription $\ddagger$

Number of prescribers writing RMG prescriptions

Extent to which access varies by geography and population subgroup

Extent to which delivery differs across the province

\section{Measure of access to RMG (CFIR domain: PharmaNet} characteristics of the intervention)

\section{Measure of access to RMG (CFIR domain: PharmaNet} characteristics of the intervention)

Measures of variability in access to RMG (CFIR PharmaNet domain: characteristics of individuals receiving and delivering the intervention)

Service provider descriptions of who is eligible Qualitative interviews, longitudinal and for RMG and models of delivery (CFIR domain: cross-sectional survey data process of implementation) 
Table 3 Continued

\begin{tabular}{lll}
\hline Outcome & Rationale and description & Data sources \\
\hline $\begin{array}{l}\text { Extent and types of barriers } \\
\text { encountered in accessing RMG }\end{array}$ & $\begin{array}{l}\text { Descriptions by people who use substances of } \\
\text { barriers encountered in accessing RMG (CFIR cross-sectional survey data } \\
\text { domains: intervention characteristics, inner and } \\
\text { outer context) }\end{array}$ & \\
$\begin{array}{ll}\text { Extent to which providers feel ready } \\
\text { and able to implement RMG }\end{array}$ & $\begin{array}{l}\text { Descriptions of perceived knowledge, skills, } \\
\text { training, organisational and system support }\end{array}$ & $\begin{array}{l}\text { Qualitative interviews, longitudinal and } \\
\text { cross-sectional survey data }\end{array}$ \\
& (CFIR domains: all) & \\
\hline
\end{tabular}

*Drug-related causes classified through ICD-9/10 codes presented in online supplemental table A1.

†Our ED data did not have complete coverage for all visits in BC. The estimated ED coverage in NACRS was $72 \%$ from 29 ED facilities submitting to NACRS.

$\ddagger$ According to both definitions 1 and 2 .

$\S \geq 5$ consecutive days of missed methadone doses or $\geq 6$ consecutive days of missed buprenorphine/naloxone doses.

ПAny missed doses within a continuous episode.

${ }^{\star *}$ OAT disengaged $\geq 3,6$ or 12 months.

DAD, Discharge Abstract Database; ED, emergency department; EQ-5D-5L, 5-Level Euro-Qol 5 Dimension; MSP, Medical Services Plan; NACRS, National Ambulatory Care Reporting System; OAT, opioid agonist treatment; PWOUD, people with opioid use disorder; RMG, Risk Mitigation Guidance.

from other routine care. We developed algorithms to identify RMG recipients by applying restrictions to our case searches using prescription data from PharmaNet including prescription history (prior RMG medication prescription records), timing (start date and duration), dispensation frequency (ie, daily) and drug type (by DIN) (box 1). From cases identified by these initial restrictions, a list of keywords was developed from the free-form codes written in the directions for use variable. A fuzzy string search of the keywords was applied. ${ }^{74}$ The fuzzy logic allowed for incomplete, truncated or commonly misspelled words to be captured in the keyword search. The keyword list was then further refined based on consultation with prescribers (who participate in the study consortium).

As this method may lead to a degree of misclassification, we developed two case definitions: one which we anticipate will have a lower sensitivity and higher specificity and an alternative with higher degree of sensitivity but lower specificity. While the definition with higher sensitivity will serve as our baseline definition of exposure to the RMG programme, the alternative will be used to assess the robustness of our results. Analyses will consider potentially differential effects according to medication type, dosage, carry length and persistence, pending sufficient statistical power.

\section{Statistical analysis}

We will conduct analysis of administrative health data in parallel with data collection and analyses of data from surveys and interviews, allowing us to identify early trends in provincial reach and impacts of RMG. Preliminary findings from interim analyses of interview and survey data will be used to guide ongoing analyses of secondary data and the refinement of tools for subsequent primary data collection.

\section{Statistical analysis for objective 1}

The impact of RMG on COVID-19 infection, non-fatal/ fatal overdose, all-cause mortality and continuity of care for SUD and other concurrent health conditions will be determined using administrative health data. The analytical approach will capture both the intent to

\section{Box 1 RMG algorithms to identify RMG exposure}

Step 1

Case definition 1: higher specificity, lower sensitivity

For each chemical type, person NOT on RMG medication in the 2 months prior to 27 March 2020 but prescribed medication after 27 March 2020 with one of the following keywords in the directions for use variable*: corona, coronavir, coronavirus, coronavirus, COVID-19, COVID-19, crisis, mitigat, mitigati, mitigatio, mitigation, pande, pandem, pandemic, pandemic withdrawal management, pprm, pwm, risk mit, risk mitigation, riskmitigation, safe supp, safe suppl, safe supply, safer supply

Case definition 2: higher sensitivity, lower specificity

For each chemical type, person NOT on RMG medication in the 2 months prior to March 27, 2020, but prescribed medication after March 27,2020 with one of the keywords in the directions for use variable as listed in definition 1 or any of the following keywords in the directions for use variable*: carries, carry, craving, deliv, delivery, dispense +deliver, dispense +delivery, dispensecarries, distancing, emerge, emergen, emergenc, emergency, emergency supply, guidan, guidanc, guideline, guidelines, illici, illicit, interim, isolatio, isolation, management, outbreak, overdo, replacement, risk, safer, suply, supply, unwit, unwitne, unwitness, unwitnessed, withd, withdr, withdra, withdraw, withdraw, withdrawa, withdrawal, witness, witnessed

Step 2

AND application of the following criteria for alcohol withdrawal and benzodiazepine cases only:

Exclusion criteria: persons with a record in MSP or PharmaNet for palliative care or cancer on 27 March-31 December 2020 and prior to first RMG prescription. Inclusion criteria: record in MSP for substance use disorder on 27 March-31 December 2020 or prior to at least one of their RMG prescriptions.

*Exclusion: 'pain' in directions for use variable. MSP, Medical Services Plan; RMG, Risk Mitigation Guidance. 
treat and per-protocol effects of RMG dispensations, combine propensity score matching and marginal structural modelling to control for baseline and time-varying potential confounders of the effect of RMG prescriptions on the study outcomes. First, we will apply propensity score matching analysis, ${ }^{75}$ with both investigator-selected covariates and a high-dimensional propensity scoring methodology using machine learning methods, ${ }^{76}$ to identify a control group of individuals who were eligible for RMG and who had characteristics similar to those receiving RMG dispensations, but did not receive RMG prescriptions at any point during study follow-up (27 March 2020-31 December 2020). The high-dimensional propensity score (hdPS) methodology is an automated data-driven approach to derive potentially important proxy variables from administrative data for inclusion in propensity score models. ${ }^{76}$ The approach represents a means of specifying an algorithm to identify a large number of proxy covariates in administrative data that are primarily collected for billing and routine administrative purposes and eliminating proxies with very low prevalence and minimal potential for causing bias. In the final hdPS step, propensity score techniques are used to adjust for the selected investigator-specified covariates and a large number of important proxy variables identified by hdPS algorithm. The extensive linked health administrative data available for this evaluation provide exceptional covariate coverage on traditional demographics, clinical history and health service use as well as additional billing information that may be incorporated into the hdPS algorithm. Preliminary figures on the number and geographical distribution of RMG recipients suggest a 5:1 match ratio will be feasible in this application.

Second, the 'per protocol' effect of RMG on outcomes will be estimated using marginal structural models, which are appropriate in situations when time-dependent variables are simultaneously confounders of the effect of interest and are predicted by previous treatment. We hypothesise exposure to RMG at time t affects timedependent variables, including engagement in treatment for SUD, mental health conditions and other medical care at time $t+1$, which in turn influences RMG exposure at time $\mathrm{t}+2$. Time-invariant and time-varying confounding at time $\mathrm{t}$ affects RMG exposure, the treatment and confounders at time $\mathrm{t}+1$. To control for time-varying confounding in the exposure-outcome relationship, inverse probability weights are estimated for each time point of the study to create a pseudo-population in which the exposure is independent of the measured confounders. The pseudopopulation is the result of assigning to each participant a weight that is inversely proportional to the participant's probability of receiving her own exposure history. Weighted estimation of the parameters of marginal structural models requires fitting several models: the structural (ie, weighted) model, the exposure model and the censoring model. Fitted values of separate regression models will thus be estimated for the numerator and the denominator of the inverse probability weights for each time point. In the numerator, a pooled logistic regression model will be estimated for the probability of RMG receipt, including only the baseline covariates, as well as the number of days on RMG in the previous week. The model used to estimate the denominator of the weight will include the baseline and time-varying covariates, in time $\mathrm{t}-1$. Similar models will be fitted to estimate the probability of censorship.

Selection of both baseline and time-varying covariates will be initially informed by a systematic review of factors associated with mortality among people who use substances. We will also draw from the results of preliminary analyses of survey and interview data, where possible, to identify other potential confounders. The modelling strategy and selected covariates will be reviewed with the consortium prior to the delivery of the final linked dataset.

We propose stratified analyses on four key population groups, including pregnant and parenting (with children aged $\leq 6$ years) women, identified via the perinatal care database; individuals with criminal justice system involvement within 12 months of RMG receipt (using linked data from the Ministry of Public Safety and the Solicitor General of BC); and a separate, stratified analysis on First Nations people, led by FNHA analysts, in accordance with FNHA data governance and the principles of ownership, control, access and possession. Additional subgroup analyses may consider RMG recipients with concurrent mental health conditions and those who have received OAT within 12 months of RMG receipt. Subgroup analyses will be considered on the basis of sufficient statistical power. Our a priori assumptions of 3\% of those with detected SUD receiving RMG prescriptions and $3 \%$ of mortality in this population over the study period indicate 2121 RMG recipients will be required to achieve $80 \%$ power to detect a $30 \%$ reduction in the hazard of the primary outcome for RMG recipients compared with control, by using a two-sided log-rank test with $5 \%$ significance level. We will adjust the parameters of the power calculation once data are collected and use this objective criterion to determine the extent of subgroup analysis we can feasibly conduct.

\section{Statistical analysis for objective 2}

The impact of RMG on uptake of public health measures to reduce the spread of COVID-19 and other behavioural and psychosocial outcomes will be determined using data from the longitudinal observational study. We will use generalised estimating equations to assess the effects of RMG on changes over time in ability to maintain physical distancing and to self-isolate as needed, substance use and related harms, income sources, health-related quality of life and mental health. A modified sandwich variance estimator will be used to correct for downward bias and improve efficiency arising from the small sample size. ${ }^{77}$ The sample size $(n=200)$ gives us $80 \%$ power to detect a clinically meaningful difference of Cohen's $\mathrm{d}=0.4$, assuming $30 \%$ attrition (final estimated $n=140$ ) ${ }^{78}$ In the analysis, attention will be paid throughout to exploring 
differences by sex/gender, rural/urban setting and substance type (ie, opioids, stimulants, benzodiazepines and alcohol). Pending sufficient sample size, distinct analyses using data from participants who self-identified as Indigenous will be designed and executed by FNHA investigators, Indigenous stakeholders and aligned with First Nations community priorities.

\section{Statistical analysis for objective 3}

Facilitators and barriers to RMG implementation will be informed through convergent analyses of quantitative and qualitative data (including data from longitudinal and cross-sectional surveys, qualitative interviews and administrative health data). This phase of the analysis is informed by the CFIR focusing on the implementation process indicators identified in table 3. Linked administrative health data will be used to identify the numbers of recipients and prescribers of RMG. Bar graphs and maps will be used to show the uptake of RMG across time and geography. Additional descriptive analyses will look at the characteristics of the population receiving RMG prescription, including sex, drug type, age, region and neighbourhood socioeconomic composition.

Qualitative interviews with people who use drugs and service providers/health planners will be transcribed verbatim, read by two researchers and analysed using NVIVO software. The initial coding framework will be based on the CFIR domains (characteristics of participants), process of implementation, intervention characteristics, and inner and outer context), and coding will proceed iteratively with the framework being refined as new themes emerge. Findings will be triangulated with descriptive analyses of quantitative data (collected via longitudinal and cross-sectional data) to examine implementation process indicators on differential understandings of eligibility and models of delivery, barriers that relate to the intervention itself, inner context, and outer context of implementation, and service provider perceptions of readiness, ability and support (table 3). Additional analyses led by FNHA investigators and Indigenous stakeholders will focus on culturally relevant issues related to RMG implementation, as reported by Indigenous people who use substances.

As a whole, this analytical strategy will provide critical insights into implementation challenges, facilitators and recommendations for improvements. Consistent with a parallel mixed-method design, this analysis will assist with the interpretation of findings generated in objectives 1 and 2, particularly variation in patterns of outcomes across measures and key subgroups.

\section{ETHICS AND DISSEMINATION}

Study activities have been developed to adhere to the TriCouncil Policy Statement: Ethical Conduct for Research Involving Humans (TCPS-2). ${ }^{79}$ All primary data will be collected by phone, online or in person, in accordance with recommended COVID-19 research practices.
Ongoing leadership provided by FNHA partners and PWLLE who identify as Indigenous on the study team will work to ensure this research continues to be guided by TCPS-2 and the Truth and Reconciliation Commission's Calls to Action related to public health, data governance and research ethics related to Indigenous peoples. ${ }^{80}$ Results will be disseminated incrementally, on an ongoing basis, through the consortium established for this study, and then published in peer-reviewed journals. Plain language summaries of results will be circulated via social media and through publication in lay magazines to reach a wider audience.

\section{Author affiliations}

${ }^{1}$ Faculty of Health Sciences, Simon Fraser University, Burnaby, British Columbia, Canada

${ }^{2}$ Centre for Health Evaluation and Outcome Sciences, St. Paul's Hospital, Vancouver, British Columbia, Canada

${ }^{3} \mathrm{BC}$ Centre for Disease Control, Vancouver, British Columbia, Canada

${ }^{4}$ Canadian Institute for Substance Use Research, Victoria, British Columbia, Canada ${ }^{5}$ School of Public Health and Social Policy, University of Victoria, Victoria, British Columbia, Canada

${ }^{6}$ British Columbia Office of the Human Rights Commissioner, Vancouver, British Columbia, Canada

${ }^{7}$ First Nations Health Authority, West Vancouver, British Columbia, Canada

${ }^{8}$ Department of Medicine, The University of British Columbia, Vancouver, British

Columbia, Canada

${ }^{9}$ Professionals for Ethical Engagement of Peers, Vancouver, British Columbia, Canada

${ }^{10} \mathrm{BC} /$ Yukon Association of Drug War Survivors, New Westminster, British Columbia, Canada

${ }^{11}$ School of Nursing, University of Victoria, Victoria, British Columbia, Canada

Acknowledgements We first acknowledge the nations whose lands on which this work is being conducted, including the $x^{\mathrm{w}} \mathrm{m} ə \theta \mathrm{k}^{\mathrm{w}} \partial \mathrm{y}^{2} \mathrm{~m}$ (Musqueam), Skwxwú7mesh (Squamish), Kwikwetlem ( $\left.k^{w} k^{w} ə \grave{\lambda} ə m\right)$ and Səlílwətal (Tsleil-waututh) in Vancouver/ Burnaby and the Songhees, Esquimalt and WSÁNEĆ peoples in Victoria. We acknowledge the support of the BC Ministries of Health and Mental Health and Addictions as well as the data stewards for each of the health administrative databases to be used in this evaluation. We also acknowledge the support of the $\mathrm{BC}$ Centre for Excellence in HIV/AIDS where much of this work was completed prior to affiliation changes for authors BN, JEM and $\mathrm{NH}$.

Contributors BN, AS and NH conceptualised the study, designed the administrative health data analysis plan and cowrote the first draft of the manuscript. $\mathrm{KU}$ and BP conceptualised the study, designed the plan for primary data collection and cowrote the first draft of the manuscript. HP and JEM designed the administrative health data analysis plan and revised the manuscript. KL, BB, LM, CB, ET and PB-M revised the manuscript. $B Z$ designed the administrative health data analysis plan. KGC designed the plan for primary data collection and revised the manuscript.

Funding This work was supported by Canadian Institutes for Health Research (CIHR, grant number 172 671) and Michael Smith Foundation for Health Research (MSFHR, grant number 18 951). KU is supported by a Canada Research Chair through CIHR. BP is supported through the Island Health Scholar in Residence funded by Island Health. BN is supported by an MSFHR Scholar award. BB is supported by a CIHR Health System Impact Fellowship. KGC is supported by an MSFHR Trainee Award and a CIHR Health Systems Impact Fellowship.

Competing interests None declared.

Patient consent for publication Not required.

Provenance and peer review Not commissioned; externally peer reviewed.

Supplemental material This content has been supplied by the author(s). It has not been vetted by BMJ Publishing Group Limited (BMJ) and may not have been peer-reviewed. Any opinions or recommendations discussed are solely those of the author(s) and are not endorsed by BMJ. BMJ disclaims all liability and responsibility arising from any reliance placed on the content. Where the content includes any translated material, BMJ does not warrant the accuracy and reliability 
of the translations (including but not limited to local regulations, clinical guidelines, terminology, drug names and drug dosages), and is not responsible for any error and/or omissions arising from translation and adaptation or otherwise.

Open access This is an open access article distributed in accordance with the Creative Commons Attribution Non Commercial (CC BY-NC 4.0) license, which permits others to distribute, remix, adapt, build upon this work non-commercially, and license their derivative works on different terms, provided the original work is properly cited, appropriate credit is given, any changes made indicated, and the use is non-commercial. See: http://creativecommons.org/licenses/by-nc/4.0/.

\section{ORCID iDs}

Bohdan Nosyk http://orcid.org/0000-0003-2513-3718

Brittany Barker http://orcid.org/0000-0002-4333-3345

Bernie Pauly http://orcid.org/0000-0002-4845-1383

\section{REFERENCES}

1 National Institute on Drug Abuse. Overdose death rates 2019, 2019. Available: https://www.drugabuse.gov/related-topics/trendsstatistics/overdose-death-rates

2 Public Health Agency of Canada. National report: apparent opioidrelated deaths in Canada, 2018. Available: https://www.canada.ca/ en/public-health/services/publications/healthy-living/national-reportapparent-opioid-related-deaths-released-march-2018.html

3 Rudd RA, Aleshire N, Zibbell JE, et al. Increases in Drug and Opioid Overdose Deaths--United States, 2000-2014. MMWR Morb Mortal Wkly Rep 2016;64:1378-82.

4 Volkow ND, Icaza MEM-M, Poznyak V, et al. Addressing the opioid crisis globally. World Psychiatry 2019;18:231-2.

5 British Columbia Coroners Service. Illicit drug overdose deaths in BC: January 1, 2009 to October 31, 2019; 2019.

6 Special Advisory Committee on the Epidemic of Opioid Overdoses. National report: apparent opioid-related deaths in Canada (January 2016 to June 2018). Ottawa: Public Health Agency of Canada, 2018.

7 British Columbia Coroners Service. Fentanyl-detected illicit drug overdose deaths: January 1, 2012 to January 31, 2019. 2019

8 Government of British Columbia. Provincial health officer declares public health emergency; 2016.

9 Laing MK, Tupper KW, Fairbairn N. Drug checking as a potential strategic overdose response in the fentanyl era. Int J Drug Policy 2018;62:59-66.

10 Government of British Columbia. Opioid use disorder: diagnosis and management in primary care; 2018.

11 British Columbia Ministry of Health. Psychiatric medications plan (plan G); 2018

12 College of Physicians and Surgeons of British Columbia. Practice standard: prescribing methadone; 2018.

13 Government of British Columbia. A guideline for the clinical management of opioid use disorder; 2017.

14 Government of British Columbia. Guidance for injectable opioid agonist treatment for opioid use disorder; 2018.

15 British Columbia Coroners Service. Illicit drug toxicity deaths in BC January 1, 2010 - September 30, 2020, 2020. Available: https:// www2.gov.bc.ca/assets/gov/birth-adoption-death-marriage-anddivorce/deaths/coroners-service/statistical/illicit-drug.pdf

16 People who need addiction services feel 'abandoned' during pandemic: CBC News, 2020. Available: https://www.cbc.ca/news/ canada/british-columbia/people-addictions-feeling-abandonedduring-pandemic-1.5527756

17 Use of Vancouver's overdose prevention sites down amid COVID-19 crisis: Global News, 2020. Available: https://globalnews.ca/news/ 6880623/vancouver-overdose-prevention-sites-covid-19/

18 Are COVID-19 distancing measures contributing to a spike in overdose deaths in the DTES?: CTV News, 2020. Available: https:// bc.ctvnews.ca/are-covid-19-distancing-measures-contributing-to-aspike-in-overdose-deaths-in-the-dtes-1.4916441

19 Don't use drugs alone, health experts say, even with physical distancing guidelines in place: CBC News, 2020. Available: https:// www.cbc.ca/news/canada/british-columbia/coronavirus-covid-19dtes-overdoses-1.5548694

20 COVID-19 pandemic pushes B.C. to move forward on safe takehome drug supply strategy: The Globe and Mail, 2020. Available: https://www.theglobeandmail.com/canada/british-columbia/articlepandemic-pushes-bc-to-move-forward-on-safe-take-home-drugsupply/

21 Vancouver overdose deaths spike amid COVID-19 crisis: CBC News, 2020. Available: https://www.cbc.ca/news/canada/british-columbia/ vancouver-overdose-deaths-spike-amid-covid-19-crisis-1.5517948
22 'Now, we are facing a global pandemic on top of a fentanylpoisoning crisis,' says BC Minister: CKPGToday.ca, 2020. Available: https://ckpgtoday.ca/2020/04/14/now-we-are-facing-a-globalpandemic-on-top-of-a-fentanyl-poisoning-crisis-says-bc-minister/

23 Becker WC, Fiellin DA. When epidemics collide: coronavirus disease 2019 (COVID-19) and the opioid crisis. Ann Intern Med 2020;173:59-60.

24 Marsden J, Darke S, Hall W, et al. Mitigating and learning from the impact of COVID-19 infection on addictive disorders. Addiction 2020;115:1007-10.

25 Slaunwhite AK, Gan WQ, Xavier C, et al. Overdose and risk factors for coronavirus disease 2019. Drug Alcohol Depend 2020;212:108047-47.

26 College of Pharmacists of British Columbia. COVID-19 Information - Prescription Refills Can Be Provided by a Pharmacist, 2020. Available: https://www.bcpharmacists.org/news/covid-19-publicinformation-prescription-refills-can-be-provided-pharmacist

27 College of Pharmacists of British Columbia. Professional Practice Policy - 71: Delivery of Opioid Agonist Treatment, 2020. Available: http://library.bcpharmacists.org/6_Resources/6-2 PPP/5003-PGP_ PPP71.pdf

28 Health Canada. Subsection 56(1) class exemption for patients, practitioners and pharmacists prescribing and providing controlled substances in canada during the coronavirus pandemic, 2020. Available: https://www.canada.ca/en/health-canada/services/ health-concerns/controlled-substances-precursor-chemicals/ policy-regulations/policy-documents/section-56-1-class-exemptionpatients-pharmacists-practitioners-controlled-substances-covid-19pandemic.html

29 Telehealth poised to take off: Georgia Straight Vancouver's News \& Entertainment Weekly, 2020. Available: https://www.straight.com/ covid-19-pandemic/telehealth-poised-to-take-off

30 'It's just exploded': patients turn to telehealth during pandemic: Daily Hive Vancouver, 2020. Available: https://dailyhive.com/vancouver/ telehealth-coronavirus-bc-doctor

31 Billing changes - COVID-19: doctors of BC, 2020. Available: https:// www.doctorsofbc.ca/news/covid-19-temporary-billing-changes

32 Province purchases Howard Johnson and Buchan hotels in Bid to create affordable housing: CBC News, 2020. Available: https://www. cbc.ca/news/canada/british-columbia/province-purchases-howardjohnson-and-buchan-hotels-in-bid-to-create-affordable-housing-1. 5626171

33 B.C. government buys Victoria hotel to house the homeless: CBC News, 2020. Available: https://www.cbc.ca/news/canada/britishcolumbia/province-hotel-housing-purchase-1.5571438

34 Government of British Columbia. Province buys American hotel for more affordable housing, 2020. Available: https://news.gov.bc.ca/ releases/2020MAH0074-001194

35 Government of British Columbia. New beds, support to help more people access addictions and recovery care, 2020. Available: https:// news.gov.bc.ca/releases/2020MMHA0034-001246

36 Government of British Columbia. Doubling youth treatment beds throughout B.C, 2020. Available: https://news.gov.bc.ca/releases/ 2020MMHA0043-001514

37 Service BC. Illicit drug overdose deaths in BC: findings of coroners investigations; 2018.

38 British Columbia Centre on Substance Use. Risk mitigation in the context of the dual public health emergencies, 2020. Available: https://www.bccsu.ca/wp-content/uploads/2020/05/RiskMitigation-in-the-Context-of-Dual-Public-Health-Emergencies-v1.6. pdf

39 Nosyk B, Guh DP, Bansback NJ, et al. Cost-effectiveness of diacetylmorphine versus methadone for chronic opioid dependence refractory to treatment. CMAJ 2012;184:E317-28.

40 Oviedo-Joekes E, Guh D, Brissette S, et al. Hydromorphone compared with Diacetylmorphine for long-term opioid dependence: a randomized clinical trial. JAMA Psychiatry 2016;73:447-55

41 Bansback N, Guh D, Oviedo-Joekes E, et al. Cost-Effectiveness of hydromorphone for severe opioid use disorder: findings from the salome randomized clinical trial. Addiction 2018;113:1264-73.

42 Tyndall M. A safer drug supply: a pragmatic and ethical response to the overdose crisis. Can Med Assoc J 2020;192:E986-7.

43 Ivsins A, Boyd J, Mayer S, et al. Barriers and facilitators to a novel low-barrier hydromorphone distribution program in Vancouver, Canada: a qualitative study. Drug Alcohol Depend 2020;216:108202.

44 Tardelli VS, Bisaga A, Arcadepani FB, et al. Prescription psychostimulants for the treatment of stimulant use disorder: a systematic review and meta-analysis. Psychopharmacology 2020;237:2233-55

45 Konstenius M, Jayaram-Lindström N, Guterstam J, et al. Methylphenidate for attention deficit hyperactivity disorder and drug 
relapse in criminal offenders with substance dependence: a 24-week randomized placebo-controlled trial. Addiction 2014;109:440-9.

46 Longo M, Wickes W, Smout M, et al. Randomized controlled trial of dexamphetamine maintenance for the treatment of methamphetamine dependence. Addiction 2010;105:146-54.

47 Galloway GP, Buscemi R, Coyle JR, et al. A randomized, placebocontrolled trial of sustained-release Dextroamphetamine for treatment of methamphetamine addiction. Clin Pharmacol Ther 2011;89:276-82.

48 Malcolm R, Myrick H, Roberts J, et al. The effects of carbamazepine and lorazepam on single versus multiple previous alcohol withdrawals in an outpatient randomized trial. J Gen Intern Med 2002;17:349-55.

49 Stuppaeck CH, Pycha R, Miller C, et al. Carbamazepine versus oxazepam in the treatment of alcohol withdrawal: a double-blind study. Alcohol Alcohol 1992;27:153-8.

50 Lucht M, Kuehn KU, Armbruster J, et al. Alcohol withdrawal treatment in intoxicated vs non-intoxicated patients: a controlled open-label study with tiapride/carbamazepine, clomethiazole and diazepam. Alcohol Alcohol 2003;38:168-75.

51 Ritola E, Malinen L. A double-blind comparison of carbamazepine and clomethiazole in the treatment of alcohol withdrawal syndrome. Acta Psychiatr Scand 1981:64:254-9.

52 Malcolm R, Ballenger JC, Sturgis ET, et al. Double-blind controlled trial comparing carbamazepine to oxazepam treatment of alcohol withdrawal. Am J Psychiatry 1989;146:617-21.

53 Kalyoncu Ö.A., Beyazyürek M, Kuru L, et al. Double-blind comparative trial with carbamazepine vs diazepam treatment of alcohol withdrawal. European Neuropsychopharmacology 1996;6:1-2.

54 Stock CJ, Carpenter L, Ying J, et al. Gabapentin versus chlordiazepoxide for outpatient alcohol detoxification treatment. Ann Pharmacother 2013;47:961-9.

55 Myrick H, Malcolm R, Randall PK, et al. A double-blind trial of gabapentin versus lorazepam in the treatment of alcohol withdrawal. Alcohol Clin Exp Res 2009;33:1582-8.

56 Baumgartner GR, Rowen RC. Transdermal clonidine versus chlordiazepoxide in alcohol withdrawal: a randomized, controlled clinical trial. South Med J 1991;84:312-21.

57 Baumgartner GR, Rowen RC. Clonidine vs chlordiazepoxide in the management of acute alcohol withdrawal syndrome. Arch Intern Med 1987;147:1223-6.

58 Patterson JF. Withdrawal from alprazolam dependency using clonazepam: clinical observations. J Clin Psychiatry 1990;51(Suppl):47-9. discussion 50-3.

59 Fluyau D, Revadigar N, Manobianco BE. Challenges of the pharmacological management of benzodiazepine withdrawal, dependence, and discontinuation. Ther Adv Psychopharmacol 2018;8:147-68.

60 Jones CM, McCance-Katz EF. Co-occurring substance use and mental disorders among adults with opioid use disorder. Drug Alcohol Depend 2019;197:78-82.

61 Fetters MD, Curry LA, Creswell JW. Achieving integration in mixed methods designs-principles and practices. Health Serv Res 2013;48:2134-56.

62 Creswell JW, Clark VLP. Designing and conducting mixed methods research. Sage publications, 2017.
63 CFIR Research Team-Center for Clinical Management Research. Consolidated framework for implementation research, 2020. Available: $\mathrm{https}: / /$ cfirguide.org/\#/guide_select

64 Damschroder LJ, Aron DC, Keith RE, et al. Fostering implementation of health services research findings into practice: a consolidated framework for advancing implementation science. Implement Sci 2009;4:50.

65 Kirk MA, Kelley C, Yankey N, et al. A systematic review of the use of the consolidated framework for implementation research. Implement Sci 2016;11:72.

66 Garner BR, Scott CK, Dennis ML, et al. The relationship between recovery and health-related quality of life. $J$ Subst Abuse Treat 2014;47:293-8.

67 Brooks R. EuroQol: the current state of play. Health Policy 1996;37:53-72

68 Kroenke K, Spitzer RL, Williams JBW, et al. Anxiety disorders in primary care: prevalence, impairment, comorbidity, and detection. Ann Intern Med 2007;146:317-25.

69 Kroenke K, Spitzer RL, Williams JBW. The patient health Questionnaire-2: validity of a two-item depression screener. Med Care 2003;41:1284-92.

70 First Nations Health Authority. First nations opioid overdose deaths rise in 2018, 2018. Available: https://www.fnha.ca/about/news-andevents/news/first-nations-opioid-overdose-deaths-rise-in-2018

71 First Nations Health Authority. Overdose data and first nations in bc: preliminary findings, 2017. Available: https://www.fnha.ca/ AboutSite/NewsAndEventsSite/NewsSite/Documents/FNHA Over doseDataAndFirstNationsInBC_PreliminaryFindings_FinalWeb_ July2017.pdf

72 Piske M, Zhou H, Min JE, et al. The cascade of care for opioid use disorder: a retrospective study in British Columbia, Canada. Addiction 2020;115:1482-93.

73 Pearce LA, Min JE, Piske M. Mortality among people with opioid use disorder during an opioid overdose public health emergency in British Columbia, Canada. BMJ 2020;368:m772.

74 Sloan S, Lafler KP. Fuzzy matching programming techniques using SAS $®$ software, 2018. Available: https://www.sas.com/content/dam/ SAS/support/en/sas-global-forum-proceedings/2018/2886-2018.pdf

75 Freemantle N, Marston L, Walters K, et al. Making inferences on treatment effects from real world data: propensity scores, confounding by indication, and other perils for the unwary in observational research. BMJ 2013;347:f6409.

76 Schneeweiss S, Rassen JA, Glynn RJ, et al. High-Dimensional propensity score adjustment in studies of treatment effects using health care claims data. Epidemiology 2009;20:512-22.

77 Wang M, Kong L, Li Z, et al. Covariance estimators for generalized estimating equations (GEE) in longitudinal analysis with small samples. Stat Med 2016;35:1706-21.

78 Brysbaert M. How many participants do we have to include in properly powered experiments? A tutorial of power analysis with reference tables. J Cogn 2019;2:16.

79 Government of Canada. Tri-Council policy statement: ethical conduct for research involving humans - TCPS 2 (2018), 2018. Available: https://ethics.gc.ca/eng/policy-politique tcps2-eptc2 2018.html

80 McNally M, Martin D, Nations F. First nations, Inuit and Métis health: considerations for Canadian health leaders in the wake of the truth and reconciliation Commission of Canada report. Healthc Manage Forum 2017;30:117-22. 